\title{
Fingerprint Matching Using Minutiae-Singular Points Network
}

\author{
Iwasokun Gabriel Babatunde \\ Department of Computer Science \\ Federal University of Technology, Akure, Nigeria \\ maxtunde@yahoo.com
}

\begin{abstract}
Biometrics have featured prominently for human verification and identification with fingerprint emerging as the dominant one. The dominance of fingerprint has been established by the continuous emergence of different forms of Automated Fingerprint Identification Systems (AFIS). In the course of performing human verification and identification, an AFIS performs fingerprint enrolment, enhancement, minutiae extraction and pattern matching. One of the challenges confronting fingerprint pattern matching is variation in image ridge orientation which often results in mismatch among images from the same source. In this paper, an algorithm for fingerprint pattern matching that addresses this problem is proposed. The algorithm uses the Euclidian and spatial relationships between the minutiae and singular points to determine the pattern matching scores for fingerprint images. Experimental study on FVC2002 fingerprint database measured the False Acceptance Rate (FAR), False Rejection Rate (FRR), Receiver Operating Characteristics (ROC) Curve, Equal Error Rate (EER) and the Average Matching Time (AMT). Analyses of the metrics obtained from the measurements revealed high adequacy level of the new algorithm at distinguishing fingerprints obtained from different sources. It is also revealed that correct matching of images from same source is heavily dependent on the quality of the images.
\end{abstract}

Keywords: Fingerprint Pattern Matching, Minutiae, FRR, FAR, ROC, EER

\section{Introduction}

Fingerprint is an impression formed through deposit of minute ridges and valleys when a finger touches a surface. Facts exist that the ridges and valleys do not change throughout lifetime no matter what happens and in a case of injury or mutilation, they reappear within a short period. The five commonly found fingerprint ridge patterns are arch, tented arch, left loop, right loop and whorl (Figure 1) [1 - 6]. Fingerprint has proved to be a very reliable human identification and verification index and has enjoyed superiority over other biometrics such as ear, nose, iris, voice, face, gait and signature [7]. The uniqueness of the ridges and valleys makes it immutable and therefore serves a strong mark for identity.

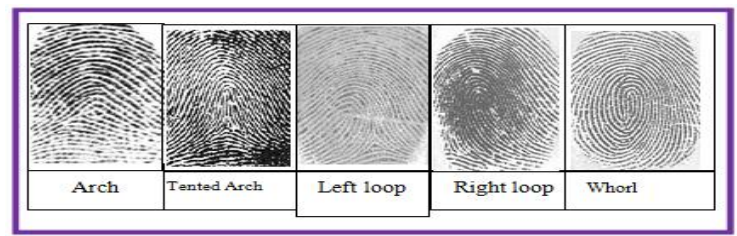

Figure 1. Types of Fingerprints Patterns 
Fingerprint pattern matching is performed when the need for ascertaining the source of a fingerprint image arises and it involves the generation of matching scores [8]. When fingerprints from the same finger are involved, the matching scores are expectedly high and low for fingerprints from different fingers. Fingerprint matching faces a number of challenges including large intra-class variations (variations in fingerprint images of the same finger) and large interclass similarity (similarity between fingerprint images from different fingers). Intraclass variations are caused by unequal pressure, orientation and translational placement with respect to the sensor. It is also caused by changes in the condition of the finger such as skin dryness and cuts. On the other hand, inter-class similarity can be large due to limited number of fingerprint pattern; namely arch, loop, and whorl [9].

Various techniques have been formulated by different authors for the matching of fingerprints. Among them is the minutiae based technique that has attracted interest from different research groups. This technique is widely adopted because fingerprint minutiae are the most unique, durable and reliable features. Minutiae-based fingerprint matching algorithm is often designed for solving problems of correspondence and similarity computation. Minutiae-based pattern matching is mostly used because forensic examiners have successfully relied on minutiae to match fingerprints for a long period of time. Minutiaebased representation is storage efficient and expert testimony about suspect identity based on mated minutiae is admissible in courts of law [9]. The latest trend in minutiae matching is to use local minutiae structures to quickly find a permissible alignment between two fingerprints and then consolidate the local matching results at a global level. This kind of matching algorithm typically consists of the steps conceptualized in Figure 2.

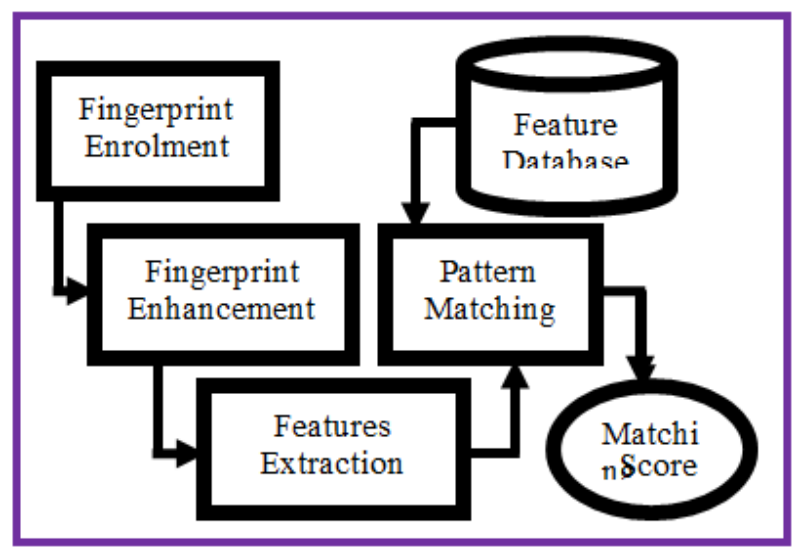

Figure 2. A Typical Fingerprint Pattern Matching Steps

The algorithm begins with fingerprint enrolment [9] followed by enhancement of the ridges and valleys. Enhancement is necessary for smooth and speedy extraction of minutiae and it involves ridge segmentation, normalization, orientation estimation, frequency estimation, filtering, binarization and thinning [10-12]. Algorithms for the extraction of minutiae points from thinned fingerprint images have been proposed in $[8,10,11,13]$. Several of these algorithms use the 8-nearest neighbors approach to extract a ridge point as a bifurcation, end, and isolated, continuing or crossing point [6]. During feature matching, a pair-wise similarity between minutiae sets of two fingerprints is computed by comparing minutiae descriptors that are invariant to rotation, size and translation [9]. Each minutia is assigned texture and minutiae-based descriptors for the correspondence problem in [14]. An alignment-based greedy matching algorithm is then used to establish the correspondences 
between minutiae. The authors in [15] proposed a novel algorithm based on global comprehensive similarity with three phases. Firstly, a minutia-simplex that contains a pair of minutiae as well as their associated textures was built to describe the Euclidean space-based relative features among minutiae. Its transformation-variant and invariant relative features were employed for the comprehensive similarity measurement and parameter estimation respectively. Secondly, the ridge-based nearest neighborhood among minutiae was used to represent the relative features. With this approach, minutiae were grouped according to their affinity with a ridge. Finally, the relationship between transformation and the comprehensive similarity between two fingerprints was modeled in terms of histogram for initial parameter estimation.

While tremendous progress has been made in plain and rolled fingerprint matching, latent fingerprint matching continues to be a difficult problem. Poor quality of ridge impressions, small finger area, and large non-linear distortion are the main difficulties in latent fingerprint matching compared to plain or rolled fingerprint matching. A system for matching latent fingerprints found at crime scenes to rolled fingerprints enrolled in law enforcement databases is proposed in [16]. Extended features, including singularity, ridge quality map, ridge flow map, ridge wavelength map, and skeleton were used. The matching module consists of minutiae, orientation field and skeleton matching. The importance of various extended features was studied and the experimental results indicate that singularity, ridge quality and ridge flow maps are the most effective features in improving the matching accuracy.

The authors in $[17,18]$ proposed a filter-based algorithm that uses a bank of Gabor filters to capture both local and global details in a fingerprint as a compact fixed length FingerCode. The verification of the algorithms revealed they are marginally inferior to minutiae-based algorithms. In this study, a minutiae-based algorithm for fingerprint pattern matching based on the Euclidian and spatial relationship among minutiae and singular point is developed. Section 2 presents the proposed fingerprint pattern matching algorithm. A case study of the benchmark FVS2002 fingerprints is presented in Section 3 while Section 4 focuses on the conclusion drawn.

\section{Proposed Fingerprint Pattern Matching Algorithm}

The new algorithm was motivated by the need to address the matching problems due to variations in image ridge orientation and size. The algorithm exploits the fact that the Euclidian and spatial relationships between the singular point and a minutia point is fixed irrespective of the directional (orientation) flow at a uniform image size as illustrated in Figure $3[12,19]$. A singular point is a core or delta point. At the core point, the ridge experiences maximum turning (change in orientation) resulting in a zero gradient (Figures 4(a) and 4(b)) while the ridge experiences a tri-dimensional change at the delta point (Figures 4(b) and 4(c)). Bifurcation and ridge end points are the commonest minutiae points with properties that uniquely describe a fingerprint image $[8,11]$. At a bifurcation point (shown with squares in Figure 4), the ridge splits into two while the ridge terminates at the end points (shown with circles in Figure 4). 


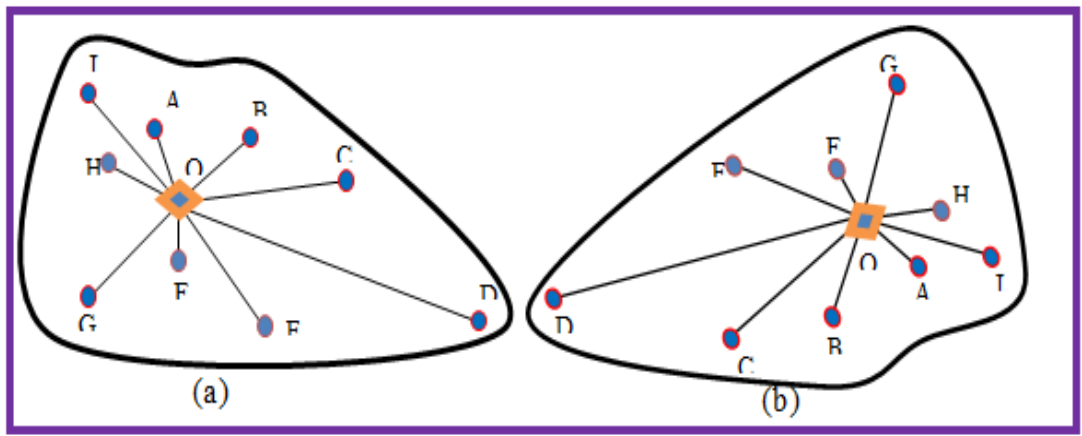

Figure 3. Interconnecting Lines between Feature and Core Points at Different Orientations

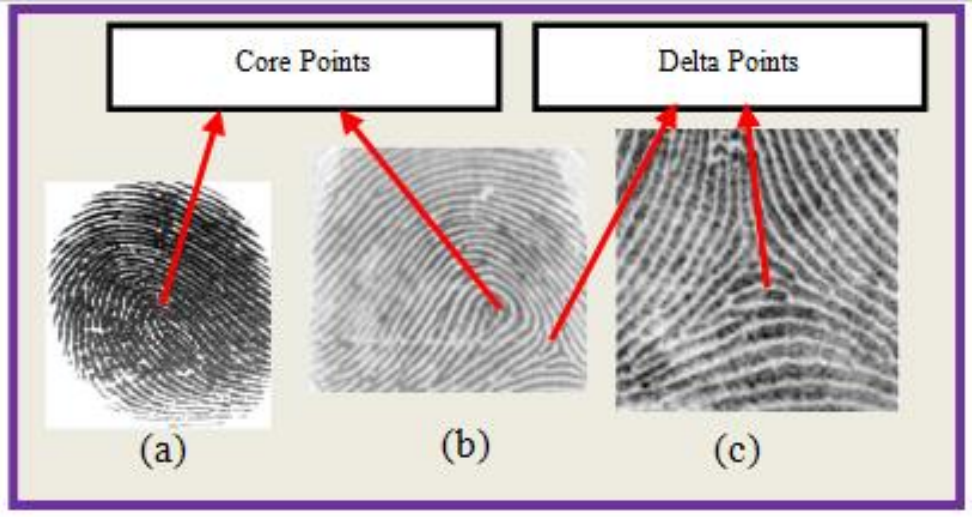

Figure 4. Fingerprint Images and their Core Points

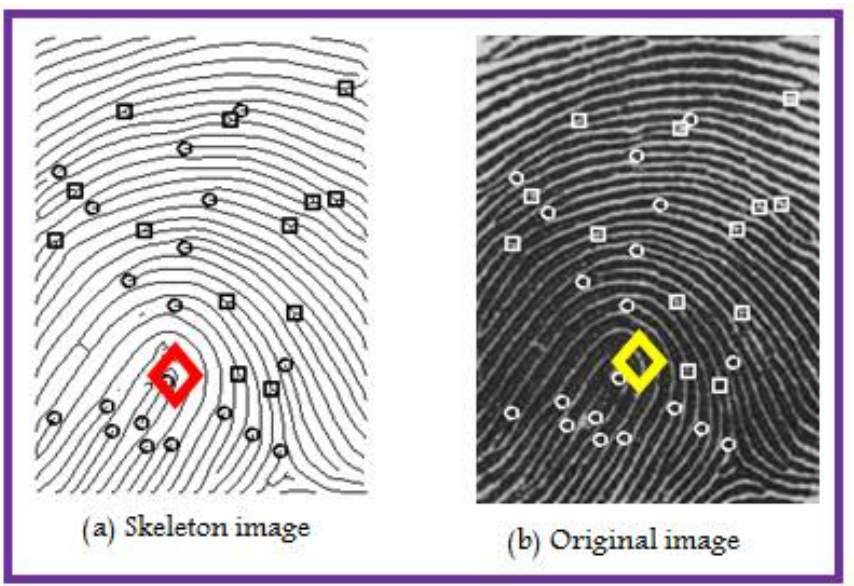

Figure 5. Feature Points for Skeleton and Original Images

The operation of the proposed algorithm is in the following phases:

a. Core point detection based on the following steps [20].

- Division of the fingerprint image, I, into blocks of size $\mathrm{N} x \mathrm{~N}$.

- Computation of the orientation (directional flow) for the center pixel $A(i, j)$ of each block. 
- The singular point characteristics for a pixel $(i, j)$ is derived as follows:

$$
\begin{gathered}
P C(i, j)=\frac{1}{\pi} \sum_{c=1}^{8} \beta_{c} \\
\beta_{c}=\left\{\begin{array}{cc}
p(c)+\pi, & \text { if } p(c) \leq-\frac{\pi}{2} \\
p(c), & \text { if } p(c)>-\frac{\pi}{2} \text { and } p(c) \leq \frac{\pi}{2} \\
p(c)-\pi, & \text { otherwise }
\end{array}\right. \\
p(c)=\left|O_{c+1}-O_{c}\right|, \quad O_{9}=O_{1}
\end{gathered}
$$

$\mathrm{O}_{1}, \mathrm{O}_{2}, \ldots, \mathrm{O}_{8}$ represent the orientations of the $3 \times 3$ neigbours of pixel (i,j) scanned in the direction shown in Figure 6.

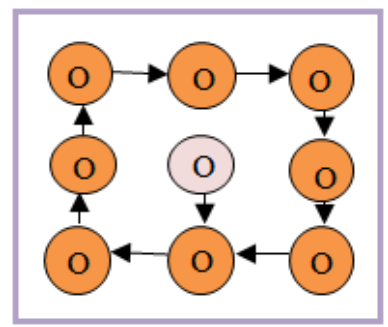

\section{Figure 6. 8 Neighbors of a Candidate Pixel}

Based on these characteristics, the core point lies between -1 and -0.5 for PC $(i, j)$ while the delta point is in the range $0.5-1.0$. False core and delta points are eliminated using a 2-step algorithm as follows:

- An average core (or delta) is calculated if there is more than one core (or delta) in a circular region with radius of 8 pixels. Given that $N$ cores (or deltas) exist in an area, $\left\{\left(\mathrm{u}_{\mathrm{i}}\right.\right.$, $\left.\left.\left.\mathrm{v}_{\mathrm{i}}\right), \mathrm{i}=1,2,3, \ldots, \mathrm{N}\right)\right\}$ then, the average core (or delta) $(u, v)$ is calculated from:

$$
\begin{aligned}
& u=\frac{1}{N} \sum_{i=1}^{N} u_{i} \\
& V=\frac{1}{N} \sum_{i=1}^{N} v_{i}
\end{aligned}
$$

- Both delta and core points are eliminated if their locations are less than or equal to 8 pixels apart

b. Extraction of all the true bifurcations and endpoints in the skeleton image (SI). The SI is obtained using the algorithms presented in [12]. The Crossing Number (CN) value for each pixel is obtained according to the formula $[8,13]$ :

$$
C N=\sum_{i=0}^{7}\left|N_{i+2}-N_{i+1}\right|, \quad N_{9}=N_{1}
$$

$\mathrm{N}_{1}, \mathrm{~N}_{2}, \ldots, \mathrm{N}_{8}$ denote the 8 neighbors of the pixel in its $3 \times 3$ neighborhood scanned in clockwise direction as follows: 


\begin{tabular}{|l|l|l|}
\hline $\mathrm{N}_{2}$ & $\mathrm{~N}_{3}$ & $\mathrm{~N}_{4}$ \\
\hline $\mathrm{N}_{1}$ & $\mathrm{~N}$ & $\mathrm{~N}_{5}$ \\
\hline $\mathrm{N}_{8}$ & $\mathrm{~N}_{7}$ & $\mathrm{~N}_{6}$ \\
\hline
\end{tabular}

As shown in Figure 7, a ridge pixel with $\mathrm{CN}$ value of 2 corresponds to a ridge ending and a $\mathrm{CN}$ value of 6 corresponds to a bifurcation.

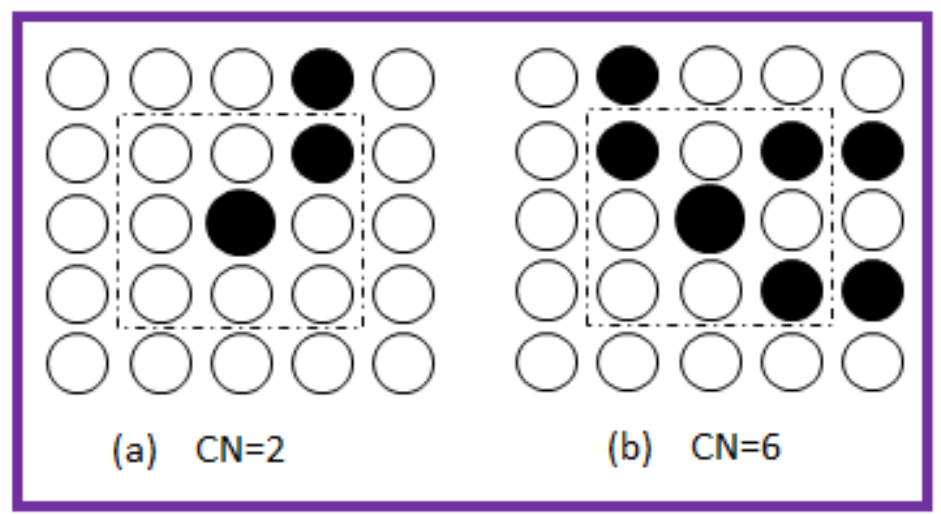

Figure 7. CN Values for Ridge Ending and Bifurcation

A minutiae validation algorithm [8] was implemented to test the validity of each extracted point. Firstly, an image $M$ of size $W x W$ centered on the candidate minutia point is created. Secondly, the central pixel of $M$ is labeled with 2 and the rest of the pixels in $M$ are initialized to zero. Subsequent steps depend on whether the candidate point is a ridge end or a bifurcation.

For a candidate bifurcation point:

- Its $3 \times 3$ neighborhood is examined in a clockwise direction and the three connecting pixels are labeled with 1.

- The three ridge pixels that are linked to the three connected pixels are also labeled with 1.

- The number of transitions from 0 to $1\left(T_{01}\right)$ along the border of image $M$ are counted in a clockwise direction. If $T_{01}=3$ (see Figure 8(a)), then the candidate minutia point is validated as a true bifurcation.

For a candidate ridge end point:

- In $M$, Label with 1 all the pixels in the 3 x 3 neighborhood of candidate point.

- Count in a clockwise direction, the number of 0 to 1 transitions $\left(T_{01}\right)$ along the border of the image. If $T_{01}=1$ (see Figure 8(b)), then the candidate minutia point is validated as a true ridge end.

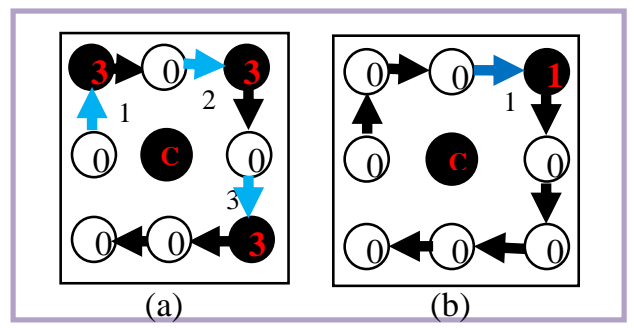

Figure 8: 0 to 1 transitions. (a) Bifurcation $\left(T_{03}=3\right)$, (b) Ridge ending $\left(T_{01}=1\right)$ 
c. The distance, $\lambda_{\mathrm{i}}$ between the $\mathrm{i}^{\text {th }}$ minutia point $P_{i}\left(a_{i}-b_{i}\right)$ and the singular point $\mathrm{M}(\rho, \sigma)$ is obtained from:

$$
\lambda_{\mathrm{i}}=\left(\left(a_{i}-\rho\right)^{2}+\left(b_{i}-\sigma\right)^{2}\right)^{0.5}
$$

d. The degree of closeness, $E_{c}$ for images $\mathrm{K}$ and $\mathrm{L}$ is obtained by using the formula:

$$
E_{c}=\sum_{i=1}^{s}(|G(i)-H(i)|) *\{G(i)\}^{-1}
$$

$\mathrm{G}(\mathrm{i})$ and $\mathrm{H}(\mathrm{i})$ represent the distance between the $\mathrm{i}^{\text {th }}$ minutia point and the core points in $\mathrm{K}$ and $\mathrm{L}$ respectively. If the number of the feature sets for $\mathrm{K}$ is lesser than or equal to the number of feature sets for $\mathrm{L}$, then $s$ is the number of the feature sets for K otherwise $s$ is the number of the feature sets for $\mathrm{L}$.

e. The correlation coefficient, $\rho$ between $\mathrm{K}$ and $\mathrm{L}$, is computed as the pattern matching score as follows:

$$
\rho=\left(1-E_{c}\right) * 10^{-2}
$$

From Equation (8), $E_{c}=0$ for exact or same images and, consequently, the matching score is $\rho=1$.

\section{Experimental Study}

The experimental study of the proposed fingerprint matching algorithm was carried out using Matlab on a Pentium $4-2.10 \mathrm{GHz}$ processor with 1.00GB of RAM in an Ms-Window Vista Home Basic Operating System environment. The study focused on analyzing the performance of the proposed algorithm on different image qualities as well as generating metric values that provided bases for comparing the proposed algorithm with some recently formulated and similar ones. Case study of FVC2002 Fingerprint Database that was jointly produced by Biometric Systems Laboratory, Bologna, Pattern Recognition and Image Processing Laboratory, Michigan and the Biometric Test Center, San Jose, United States of America was carried out. The database consists of four datasets DB1, DB2, DB3 and DB4 and its summary is presented in Table 1 [21].

Table 1. Details of FVC2002 Fingerprint Database

\begin{tabular}{|l|l|lll|l|l|}
\hline $\begin{array}{l}\text { Data- } \\
\text { base }\end{array}$ & Sensor Type & Image size & Number & Resolution \\
\hline DB1 & Optical Sensor & $\begin{array}{l}388 \times \quad 374 \\
\text { Kpixels })\end{array}$ & $(142$ & $10 \times 8$ & 500 dpi \\
\hline DB2 & Optical Sensor & $\begin{array}{l}296 \times 560 \\
\text { Kpixels })\end{array}$ & $(162$ & $10 \times 8$ & 569 dpi \\
\hline DB3 & Capacitive Sensor & $\begin{array}{l}300 \times 300 \\
\text { Kpixels })\end{array}$ & $(88$ & $10 \times 8$ & 500 dpi \\
\hline DB4 & SFinGe v2.51 & $\begin{array}{l}288 \times 384 \\
\text { Kpixels })\end{array}$ & $(108$ & $10 \times 8$ & About 500 dpi \\
\hline
\end{tabular}


Each dataset comprises of a set of 8 impressions from each of 10 different fingers. FRR, FAR, ROC Curve, EER and AMT were the indicators measured. These indicators were chosen based on their global acceptability for evaluating the performance of biometric pattern matching systems [9]. FRR is the rate of occurrence of a scenario of two fingerprints from same finger failing to match (the matching score falling below the threshold) while FAR is the rate of occurrence of a scenario of two fingerprints from different fingers found to match (matching score exceeding the threshold). An ROC curve depicts the plot of FRR against FAR for all possible matching thresholds and measures the overall performance of the system. Each point on the curve is a particular decision threshold. In the ideal case, both FAR and FRR should be zero and the genuine and imposter distributions should be disjoint. In such case, a 'reasonable' ROC curve is a step function at the zero FAR. On the other extreme, if the genuine and imposter distributions are the same, then the ROC curve is a line segment with a slope of $45^{\circ}$ with an end-point at zero FAR. In practice, the ROC curve behaves in between these two extremes [9]. For each matching threshold, $i$, EER is the value at which FAR ( $i)$ and FRR ( $i$ ) are equal. For each dataset, FRR was measured by matching fingerprints obtained from the same finger while FAR was measured by matching every fingerprint image of each finger with all fingerprints of the other fingers.

Some of the results from the estimation of the core and delta points by the singular point detection algorithm are shown in Figures 9(a-c). The estimated orientation fields for these images are shown in Figures 9(d-f) while Figures 10(a-c) present the extracted endpoints (represented in circles) and bifurcation points (represented in squares) on the skeleton images. The network of connections from the singular point to each of the minutiae points in the images are shown in Figure 10(d-f). The results presented in Figures 9 and 10 demonstrate accurate extraction and estimation of the singular points and the orientation field respectively. Figure 10(d-f) also present the chains of connection between the singular and the minutiae points.

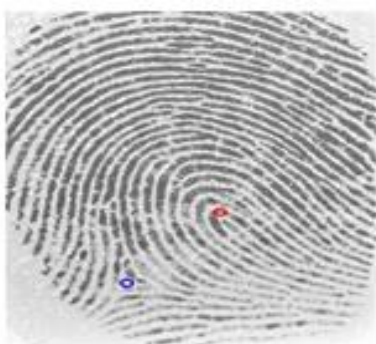

(a)

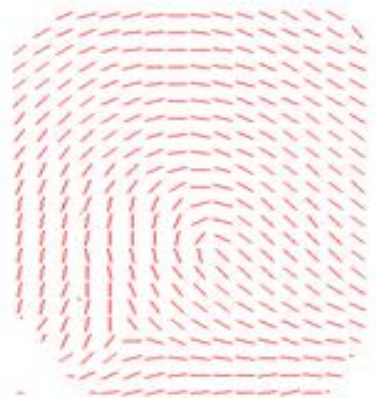

(d)

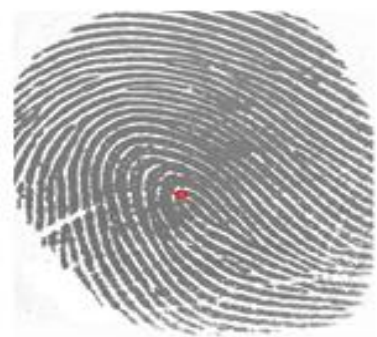

(b)

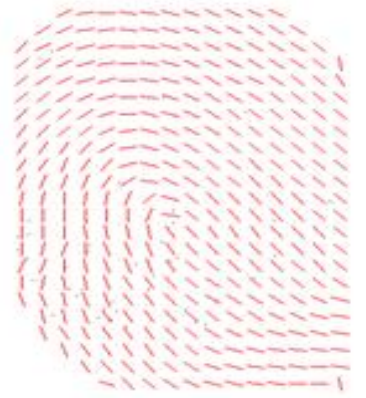

(e)

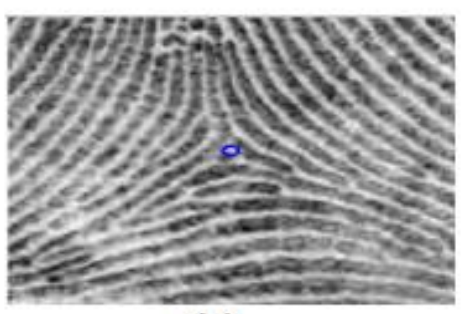

(c)

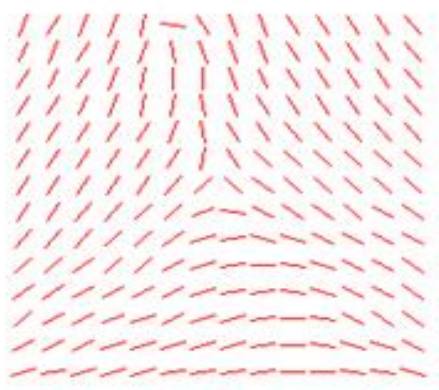

(f)

Figure 9. Fingerprint Images and their Extracted Singular Points and Orientation Field 


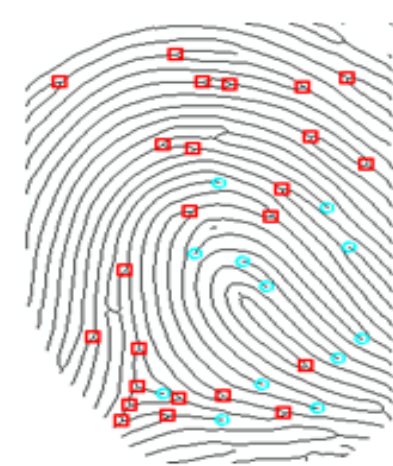

(a)

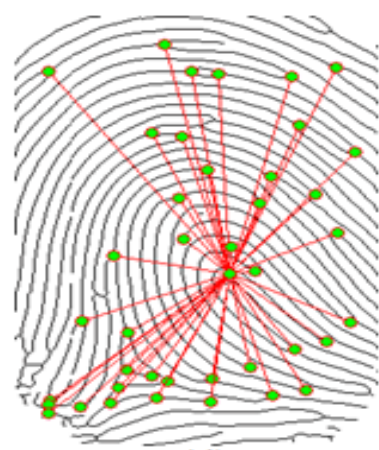

(d)

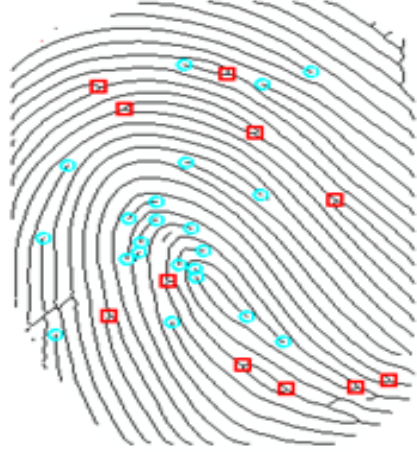

(b)

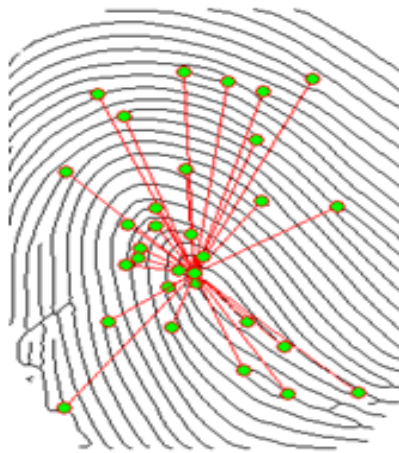

(e)

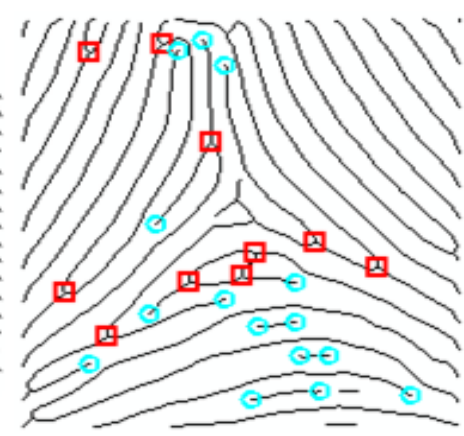

(c)

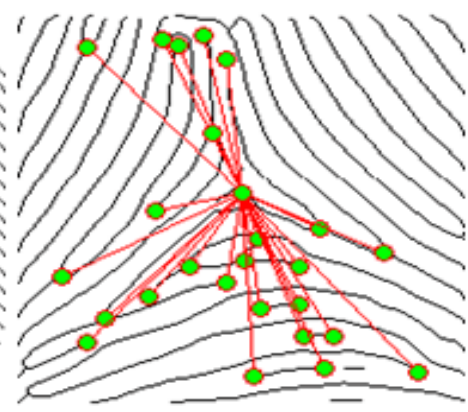

(d)

\section{Figure 10. Connections between the Extracted Minutiae and the Singular Points}

During matching, for image with core and delta points, priority was given to the core point and the FAR and FRR results obtained at a threshold value for the four datasets are shown in Table 2.

\section{Table 2. FAR and FRR Values in Percentage (\%) for the Four Datasets}

\begin{tabular}{|l|l|l|l|l|}
\hline Statistics & DB1 & DB2 & DB3 & DB4 \\
\hline FAR & 0 & 0 & 0 & 0 \\
\hline FRR & 10.23 & 7.85 & 5.51 & 7.47 \\
\hline
\end{tabular}

Table 2 reveals that for each dataset, FAR of $0 \%$ was generated which indicates adequacy and the ability of the new algorithm to identify fingerprint images from different fingers. However, the obtained FRR values of $10.23 \%$, $7.85 \%$, 5.51\% and $7.47 \%$ present the failure rate of the algorithm for matching fingerprints from the same finger in datasets DB1, DB2, DB3 and DB4 respectively. Variations in the failure rates are attributed to differences in pressure and contact area during fingerprint enrolment [9]. The differences forced images enrolled from the same finger to show differences in quality, contrast and noise levels and consequently, different matching scores for different pairs of fingerprints of same finger.

Visual inspection of fingerprints in the four datasets confirms this performance trend. The inspection reveals best image quality for dataset DB3 followed by DB4, DB2 and DB1. This is premised on the fact that images in dataset DB3 exhibit greatest form of clarity of ridges and valleys and very rare noise levels. Based on these, same source images in dataset DB3 
experienced best enhancement and extraction of highest number of valid minutiae points with similar characteristics. The highest FRR value of $10.23 \%$ for dataset DB1 is attributed to poor enhancement and generation of significant number of artifacts. Artifacts are foreign ridges and valleys introduced into the image inform of cross over, hole or spike structures during the enhancement process [8]. The artifacts mislead the feature extraction algorithm into extraction from images from same finger, disparate and high number of false minutiae (ridge ending and bifurcation) with unequal properties. The inequality in the minutiae properties explained the highest false rejection rate. Visual inspection of the 80 fingerprint images in dataset DB4 also reveals better ridge and valley structures when compared with images in datasets DB1 and DB2. This buttressed the lower FRR for dataset DB4 than for datasets DB1 and DB2. In the overall, the proposed pattern matching algorithm returned an average FRR value of $7.76 \%$ over the four datasets representing the extent to which the algorithm failed to match same source fingerprints from their collections.

The trend of the average matching times in seconds for FRR and FAR over the four datasets is presented in Figure 11. The higher FAR matching times recorded for all datasets is attributed to matching of fingerprints from multiple sources which outnumbered same source fingerprints used for FRR. Dataset DB3 has the lowest FRR average matching time of 0.42 second followed by DB4, DB2 and DB1 with FRR average matching time of $0.51,0.61$ and 0.72 second respectively. DB3 also recorded the lowest FAR average matching time of 0.63 second followed by DB4, DB2 and DB1 with average matching time of $0.75,1.01$ and 1.01 second respectively. The lowest FRR and FAR average matching times for dataset DB3 implies that its fingerprint images have fewest number of minutiae points when compared to other datasets and consequently, smallest number of computations (matching). Similarly, the highest FRR and FAR average matching times for dataset DB1 indicates highest number of minutiae points for its images and consequently, highest number of computations.

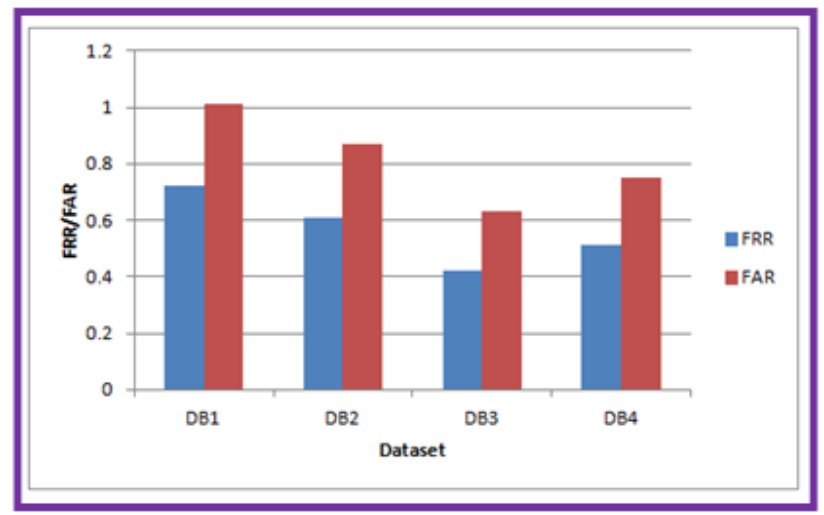

\section{Figure 11. Column Chart of the FRR Matching Completion for the Four Datasets}

Figure 12 presents the obtained ROC Curves for the four datasets. The curves justify the results obtained for FAR and FRR having recorded the highest and least FRR at zero FAR for dataset DB3 and DB1 respectively. 


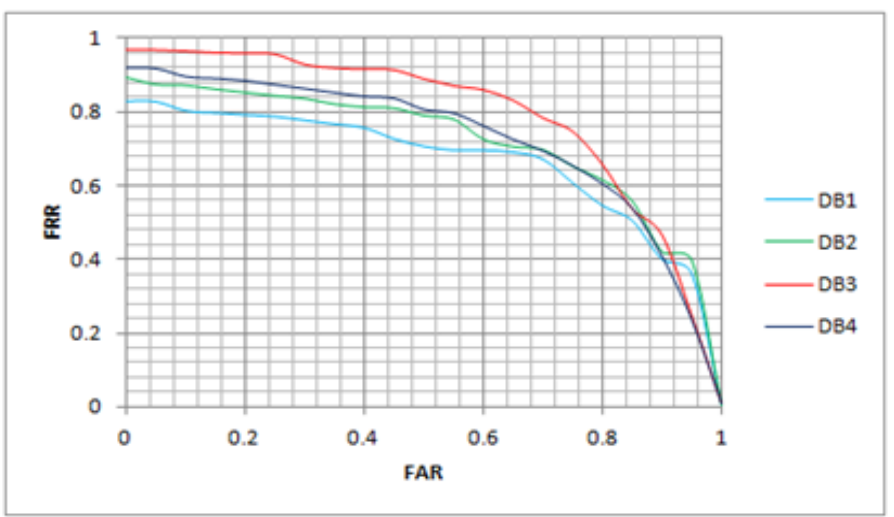

Figure 12. ROC Curves for the Four

Equal Error Rates (EERs) were also generated to determine the best single description of the error. The lower the value of EER, the lower is the error rate and the greater is the adequacy of the algorithm. For datasets DB1, DB2, DB3 and DB4, EER points $(0.2657$, $0.00129),(0.2612,0.00214),(0.2005,0.00103)$ and $(0.2356,0.0017)$ were recorded respectively as shown in Figure 13. The implication of these results is that for the thresholds $0.2657,0.2612,0.2005$ and 0.2356 there are guarantees of the same FAR and FRR error rates of $0.00129,0.00214,0.00103$ and 0.0017 for the algorithm on datasets DB1, DB2, DB3 and DB4 respectively. It also implied that $1.29,2.14,1.03$ and 1.7 out of every 1000 impostors (or genuine) attempts will succeed (or fail) based on dataset DB1, DB2, DB3 and DB4 respectively. The obtained FRR and FAR values for four different algorithms are presented in Table 3. The algorithms presented in [22-24] were selected for comparison because they ranked among recently formulated fingerprint matching algorithms and their evaluations are also based on FVC2002 fingerprint database.

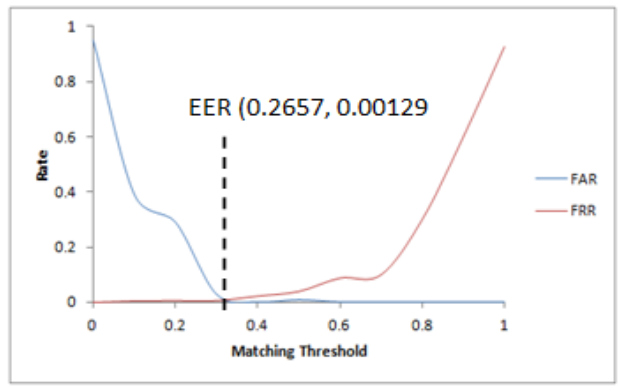

(c) Dataset DB1

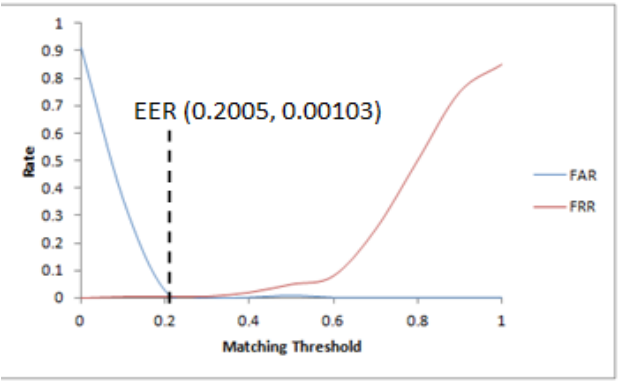

(b) Dataset DB3

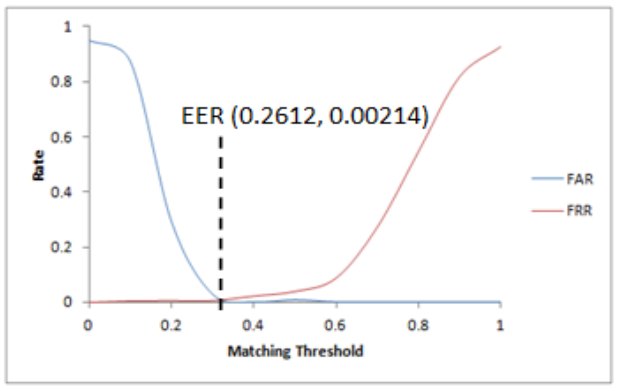

(d) Dataset DB2

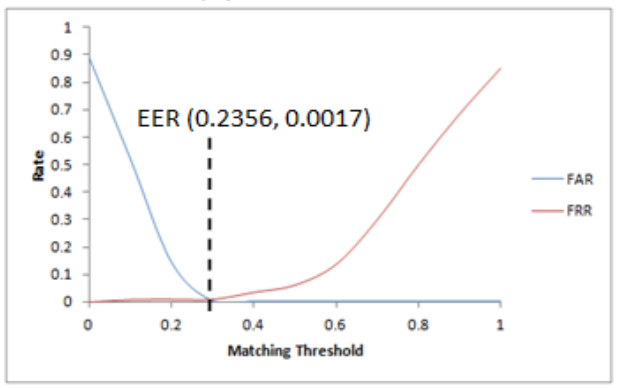

(a) Dataset DB4

Figure 13. ERR Graph for FVC2002 Fingerprint Database 
Table 3. FAR and FRR for Different Algorithms

\begin{tabular}{|l|l|l|l|l|l|l|l|l|}
\hline & \multicolumn{2}{|c|}{ Ref. [22] } & \multicolumn{2}{c|}{ Ref. [23] } & \multicolumn{2}{c|}{ Ref. [24] } & \multicolumn{2}{l|}{ Current Study } \\
\hline Dataset & FRR & FAR & FRR & FAR & FRR & FAR & FRR & FAR \\
\hline DB1 & 52.58 & 0 & 89.3 & 1.7 & 23.07 & 0 & 10.23 & 0 \\
\hline DB2 & 50.03 & 0 & 88.6 & 3.7 & 19.91 & 0 & 7.85 & 0 \\
\hline DB3 & 73.75 & 0 & 91.2 & 2.4 & 16.68 & 0 & 5.51 & 0 \\
\hline DB4 & 65.24 & .015 & 81.3 & 0.9 & 17.09 & 0.01 & 7.47 & 0 \\
\hline
\end{tabular}

Table 3 features the original values presented in $[22,24]$ while the algorithm proposed in [23] was implemented based on the conditions specified for the new algorithm to obtain the stated values. The superior performance of the new algorithm over the three algorithms is clearly exhibited with its lowest FRR values for all the datasets. In addition, it is the only algorithm with an FAR value of zero for all the datasets. Figures 14 and 15 present obtained FRR and FAR computations times in seconds in $[23,24]$ and the current study. The original algorithm proposed in [23] was also implemented under equal condition of experiments to obtain the stated values. For all the datasets, the proposed algorithm exhibited lower computation times, which confirms its superiority in operational speed.

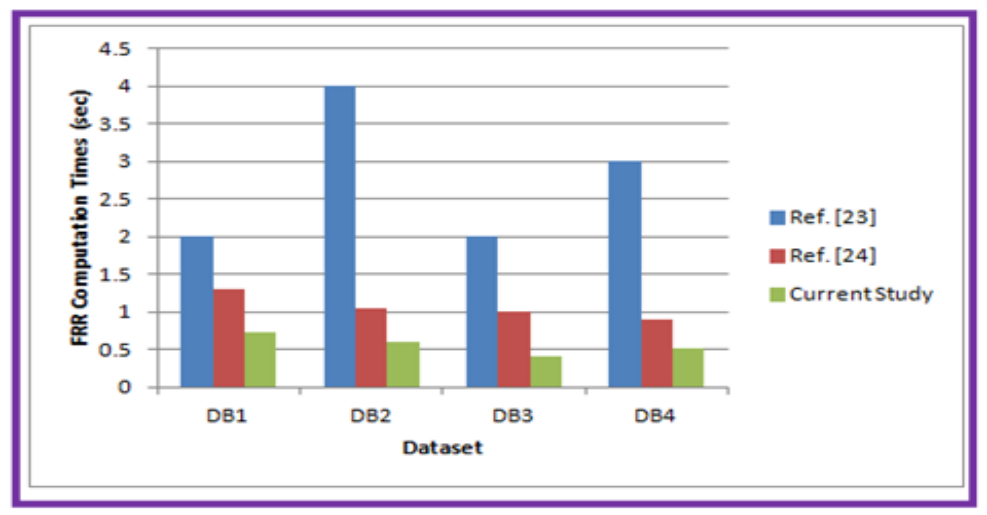

Figure 14. Computation Time for FRR Values for Different Fingerprint Matching Algorithms

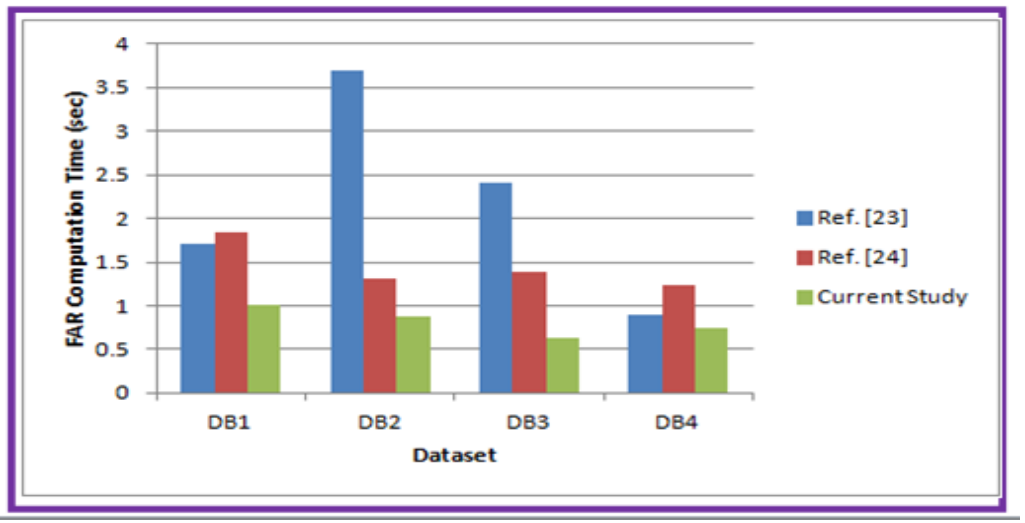

Figure 15. Computation Time for FAR Values for Different Fingerprint Matching Algorithms 
Figure 16 illustrates the average FRR based on the data presented in Tables 3. Similarly, Figure 17 presents the average FRR and FAR computation times over the four datasets. Visual inspection of the two Figures reveals best performances for the proposed algorithm.

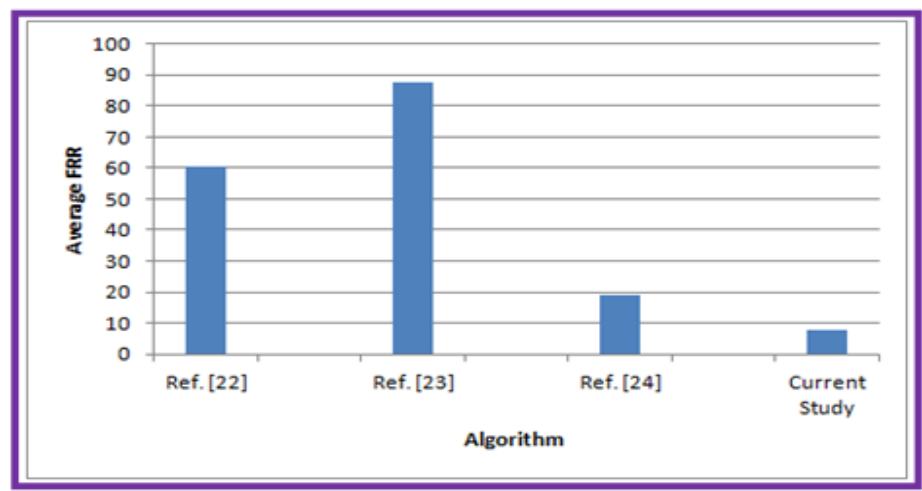

Figure 16. Colum Chart of Average FRR Values for Different Fingerprint Matching Algorithms over the Four Datasets

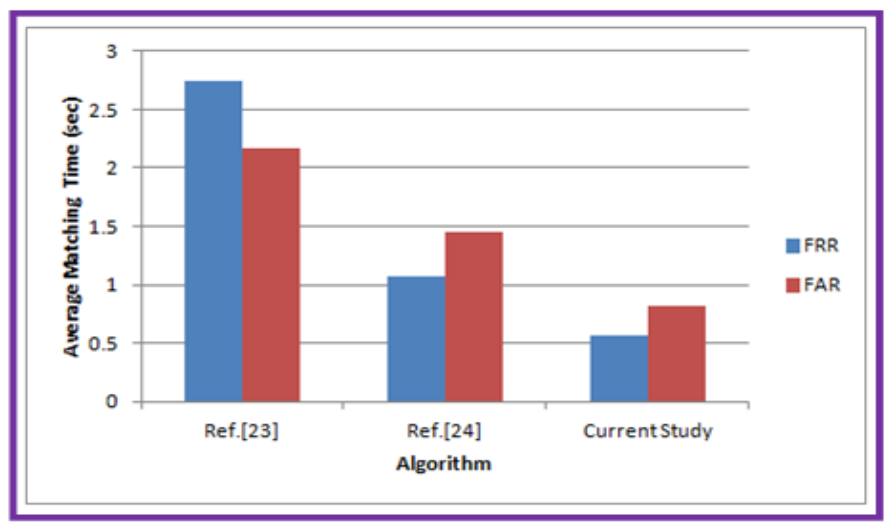

Figure 17. Colum Chart of Average Computation time for FRR and FAR Values for Different Fingerprint Matching Algorithms over the Four Datasets

\section{Conclusion and Future Works}

The implementation of a new minutiae-based fingerprint pattern matching algorithm has been presented. The algorithm uses the Euclidian and spatial characteristics of the minutiae and the core points to determine the similarity score for two fingerprint images. The obtained results showed the effectiveness of the algorithm at distinguishing fingerprints from different sources with average FAR of $0 \%$. The algorithm yielded different FRR values for the used datasets due to unequal corruption and noise levels. The first dataset is mostly affected with FRR values of $10.23 \%$ while the third dataset is least affected with FRR value of $5.51 \%$. A comparison of the obtained results with what obtained for some recently formulated algorithms over the same datasets revealed superior performance for the proposed algorithm. The implication of this superiority is that the new algorithm is more suitable for reducing the error rates and improving on the performances of the existing AFIS. Future research direction aims at the optimization of the proposed algorithm for further reduction in the FRR values and the computation times. 


\section{References}

[1] W. G. Eckert, "Introduction to Forensic Science", New York: Elsevier, (2006).

[2] "FIDIS, Future of Identity in the Information Society", Elsvier Inc., (2006).

[3] D. Salter, "Thumbprint - An Emerging Technology", Engineering Technology, New Mexico State University, (2006).

[4] J. Wayman, D. Maltoni, A. Jain and D. Maio, "Biometric Systems", Springer-Verlag London Limited, (2005).

[5] O. C. Akinyokun and E. O. Adegbeyeni, "Scientific Evaluation of the Process of Scanning and Forensic Analysis of Thumbprints on Ballot Papers", Proceedings of Academy of Legal, Ethical and Regulatory Issues, vol. 13, no. 1, (2009), New Orleans.

[6] L. Yount, "Forensic Science”, From Fibres to Thumbprints' Chelsea House Publisher, (2007).

[7] C. Roberts, "Biometrics", (http://www.ccip.govt.nz/ newsroom/informoation-notes/2005/ biometrics.pdf).

[8] G. B. Iwasokun, O. C. Akinyokun, B. K. Alese and O. Olabode, "A Modified Approach to Crossing Number and Post-Processing Algorithms for Fingerprint Minutiae Extraction and Validation", IMS Manthan International Journal of Computer Science and Technology, Indian, vol. 6, Issue 1, (2011), pp. 1-9.

[9] A. K. Jain, F. Jianjiang and N. Karthik, "Fingerprint Matching", IEEE Computer Society, (2010), pp. 36-44.

[10] T. Raymond, "Fingerprint Image Enhancement and Minutiae Extraction", PhD Thesis Submitted to School of Computer Science and Software Engineering, University of Western Australia, (2003), pp. 21-56, www.peterkovesi.com/studentprojects/ raymondthai/ RaymondThai.pdf. Accessed 16/05/2009.

[11] L. Hong, Y. Wau and A. J. Anil, "Fingerprint image enhancement: Algorithm and performance evaluation", Pattern Recognition and Image Processing Laboratory, Department of Computer Science, Michigan State University, (2006), pp. 1-30.

[12] G. B. Iwasokun, O. C. Akinyokun, B. K. Alese and O. Olabode, "Fingerprint Image Enhancement: Segmentation to Thinning", International Journal of Advanced Computer Science and Applications (IJACSA), Indian, vol. 3, no. 1, (2012).

[13] G. B. Iwasokun, O. C. Akinyokun, B. K. Alese and O. Olabode, "Adaptive and Faster Approach to Fingerprint Minutiae Extraction and Validation", International Journal of Computer Science and Security, Malaysia, vol. 5, Issue 4, (2011), pp. 414-424.

[14] F. Jianjiang, "Combining minutiae descriptors for fingerprint matching”, Elsevier Pattern Recognition, vol. 41, (2008), pp. $342-352$.

[15] H. Yuliang, T. Jie, L. Liang, C. Hong and Y. Xin, "Fingerprint Matching Based on Global Comprehensive Similarity", (2005), Available online at http://www.fingerpass.net/downloads/papers/Fingerprint\%20Matching\%20Based\%20on\%20Global\%20Com prehensive\%20Similarity.pdf. Accessed 21/08/2011.

[16] K. J.Anil and F. Jianjiang, "Latent Fingerprint Matching", IEEE Transactions on Pattern Analysis and Machine Intelligence, vol. 33, no. 1, (2011), pp. 88-100.

[17] A. K. Jain, S. Prabhakar, L. Hong and S. Pankanti, "Filterbank-Based Fingerprint Matching", IEEE Transactions on Image Processing, vol. 9, no. 5, (2000).

[18] A. K. Jain, L. Hong, S. Pankanti and R. Bolle, "An identity authentication system using fingerprints", Proceedings of IEEE, vol. 85, no. 9, (1997), pp. 1365-1388.

[19] G. B. Iwasokun, "Development of a Hybrid Platform for the Pattern Recognition and Matching of Thumbprints", PhD Thesis, Department of Computer Science, Federal University of Technology, Akure, Nigeria, (2012).

[20] A. C. López, R. L. Ricardo and R. C. Queeman, "Fingerprint Pattern Recognition", PhD Thesis, Electrical Engineering Department, Polytechnic University, Mexico, (2002).

[21] D. Maio, D. Maltoni, R. Cappelli, J. L. Wayman and A. K. Jain, "FVC2002: Second Fingerprint Verification Competition", in 16th International Conference on Pattern Recognition, (2002), pp. 811 - 814.

[22] A. J. Perez-Diaz and I. C. Arronte-Lopez, "Fingerprint Matching and Non-Matching Analysis for Different Tolerance Rotation Degrees in Commercial Matching Algorithms", Journal of Applied Research and Technology, vol. 8, no. 2, (2010), pp. 186-199.

[23] P. Peer, "Fingerprint-Based Verification System A Research Prototype", IWSSIP 2010 - 17th International Conference on Systems, Signals and Image Processing, (2010), pp. 150-153.

[24] T. Li, C. Liang and K. Sei-ichiro, "Fingerprint Matching Using Dual Hilbert Scans", SITIS, (2009), pp. 553559. 